\title{
Noise induced effects in overdamped Josephson junction in the presence of colored noise
}

\author{
G. Augello*, , D. Valenti* and B. Spagnolo* \\ ${ }^{*}$ Dipartimento di Fisica e Tecnologie Relative and CNISM, Group of Interdisciplinary Physics, \\ Università di Palermo Viale delle Scienze, I-90128 Palermo, Italy \\ †augello@gip.dft.unipa.it
}

\begin{abstract}
We analyze the role of the correlated fluctuations, with a correlation time $\tau_{c}$, in the dynamics of an overdamped Josephson junction in the presence of a periodic driving signal.
\end{abstract}

Keywords: Josephson junction, mean switching time, colored noise

PACS: $85.25 . \mathrm{Cp}, 74.40 .+\mathrm{k}$

\section{MODEL AND RESULTS}

The dynamics of an overdamped Josephson junction is described by the following Langevin equation:

$$
\frac{d \phi}{d t}=-\omega_{c} \frac{d U(\phi)}{d \phi}-\omega_{c} z(t)
$$

where $\phi$ is the order parameter, $\omega_{c}$ is the characteristic frequency of the Josephson junction, $U(\phi)$ is the potential profile and $z(t)$ is the Ornstein-Uhlenbeck process with correlation time $\tau_{c}$. We analyzed this dynamical equation by numerical simulation. We found that the positions both of the minimum of resonant activation (RA) and maximum of noise enhanced stability (NES) depend on the value of $\tau_{c}$. We found a wide range in
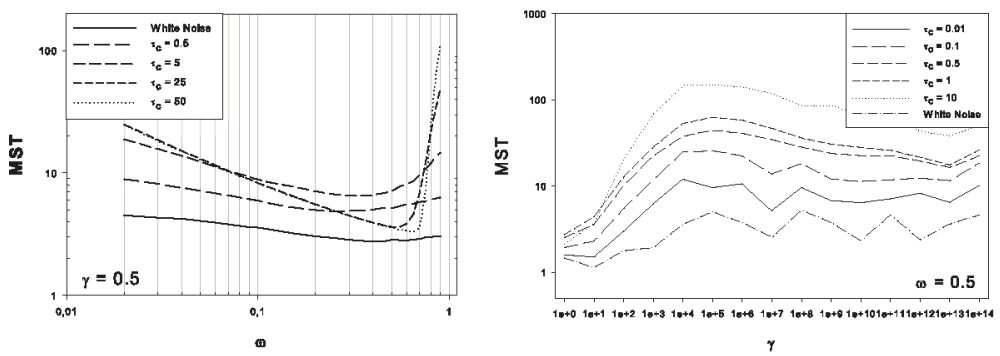

FIGURE 1. Figure on the left: curves of the MST vs $\omega$ for different $\tau_{c}$ and for $\gamma=0.5$. Figure on the right: curves of the MST vs $\gamma$.

the pulsation frequency, $0.3<\omega<0.8$, in which a non-monotonic behavior of the MST is present (figure 1, on the left). The NES phenomenon is increased by the colored noise: higher values of the $\tau_{c}$ correspond to higher value of the MST (figure 2, on the right).

CP965, Complexity, Metastability and Nonextensivity-CTNEXT 07, edited by S. Abe, H. Herrmann, P. Quarati, A. Rapisarda, and C. Tsallis (C) 2007 American Institute of Physics 978-0-7354-0481-6/07/\$23.00 